\title{
Cognitive Radar: A Way Forward
}

\author{
Dr. Michael Wicks ${ }^{1}$ \\ Air Force Research Laboratory, Sensors Directorate, USA ${ }^{I}$ \\ michael.wicks@us.af.mil
}

\begin{abstract}
The desire to autonomously anticipate, find, fix, track, target, engage, and assess, anything, anytime, anywhere (AF2T2EA4) in spectrally dense environments will require changes to how we build, modify, and deploy radar and radio frequency systems. These spectrally dense environments coupled with the twenty-first century threat are already causing havoc throughout the world.
\end{abstract}

\section{Why Cognitive Processing}

Figure 1 notionally illustrates that as technology has progressed, the availability of frequency spectrum for radar sensor operations has been severely compromised, available frequency bands have been diminished and increasingly dispersed. Line thickness in Figure 1 is indicative of radiated power limitations due to regulations, while time increments are a function of position and/or operational schedules of other radiators in space, time, and bandwidth/frequency.

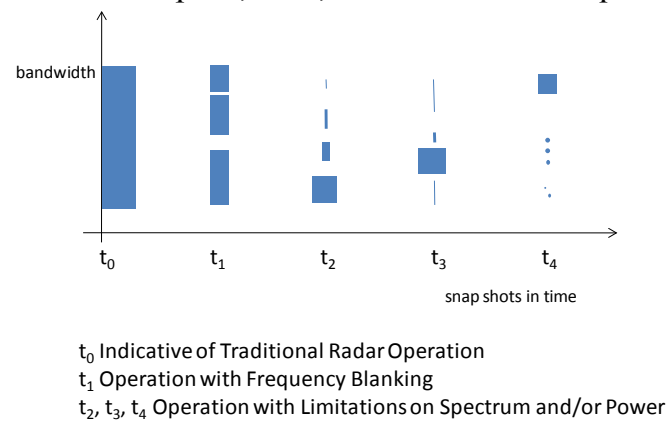

Fig. 1. Radar frequency bands and limitations

This unique issue of spectrum crowding and steadily increasing radar requirements cannot be addressed by traditional modes of operation. Future systems will require the ability to anticipate the behavior of radiators in the operational environment. This in turn leads to the need for critical and new found methodologies based upon cognition as an enabling technology.

If the USAF is going to anticipate actions before they occur, generate situational awareness, and directly support the delivery of effects - then intelligent aggregation of sensor data,

\footnotetext{
${ }^{1}$ The author wishes to acknowledge insightful collaborations with Drs Gerard Capraro, Richard Schneible and Mr. Walt Szczepanski.
}

information sources, and knowledge in real-time is required. Achieving the effects of cognition is essential to this goal. In this paper, we focus on aspects of cognition in a solitary radar system, for the purpose of spectrum allocation, recognizing that multi-sensor systems will benefit most from this technology.

In light of this spectrum encroachment, intelligent spectrum utilization coupled with agile waveform selection and signal processing in futuristic radars has become a necessity. We have formulated a cohesive plan to address these issues from a sensor and information perspective and have generated a way forward - Cognitive Radar. The Cognitive Radar will achieve the effects of cognition in the context of radar operation using perception, memory and judgment. The Cognitive Radar will have the equivalent effect of cognition, i.e., ability to "reason," without developing "consciousness" through the effective use of radar signal/data/image/track processing; waveform generation, timing and control; platform placement; and feedback for further adaptation and enhanced control. We must make Cognitive Radar a reality in order to anticipate and counter emerging threats before they attack in today's dynamic and ever changing environment, especially in urban centers where spectrum is most severely congested.

Figure 2 illustrates the essential drivers of Cognitive Radar for operation in spectrally congested environments - spectrum limitation, autonomous operation and improved performance all drive the need for Cognitive Radar.

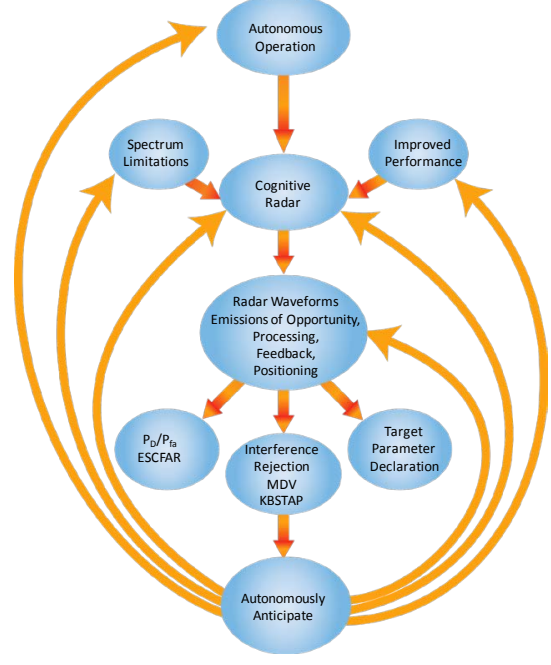

Fig. 2. Technology Drivers in Cognitive Radar

A confluence of factors must come together to meet the requirements identified above. The need for autonomous operations within the context of remote sensing demands that 
both platform and radar be employed intelligently and effectively. Spectral awareness is a critical component of Cognitive Radar. In order to operate dynamically within geographically and temporally defined spectrum limitations, a number of challenges in spectrum management must be overcome. Key to success is geographical awareness for operations in rural, urban and theater-wide environments. Knowledge sources include geospatial information and products, imagery, map data, DTED, DFAD, LULC, etc. Radar data from multiple scans and earlier flights also provides information on targets, clutter and other active sources of interference. The key is to intelligently use these data/information/knowledge sources to realize a field deployable and successful Cognitive Radar.

A brief history of intelligent radar development at the US Air Force Research Laboratory (AFRL) leading to the concept of Cognitive Radar is presented in Section III. The desire to autonomously anticipate, find, fix, track, target, engage, and assess, anything, anytime, anywhere (AF2T2EA4) in spectrally dense environments will require changes to how we build, modify, and deploy radar and sensor systems. AFRL is attacking these issues from a sensor and information perspective and has generated a way forward. The use of intelligent waveform selection and signal processing in futuristic radars is necessary. The twenty-first century threat is causing havoc throughout the world. We must make Cognitive Radar a reality in order to anticipate and counter these emerging threats before they attack in today's dynamic and ever changing environments, especially in urban centers where spectrum in most severely congested.

\section{HiSTORY OF INTELLIGENT RADAR DEVELOPMENT, A LABORATORY PERSPECTIVE}

As radar systems have been tasked to address changing threats in more severe environments, the associated signal processing has evolved from deterministic to adaptive, to intelligent, and in the future may evolve to cognitive. Figure 3 presents an early radar block diagram, with limited deterministic signal processing. Its performance would have been limited to the detection of relatively fast targets in benign environments. Adaptive processing allowed radars to work in more severe environments and against slower targets. This adaptive processing takes place in the filter and detector stages of processing illustrated in the block diagram. But successful adaptive processing historically assumed that the interference is homogeneous. Intelligent processing has been developed to overcome this homogeneity constraint and is described in section 3B.

Figure 4 illustrates the evolution of intelligent signal processing at AFRL. For example, early work in resource allocation [1] focused on the application of traditional interference suppression techniques in existing radars. Simple examples included the use of coherent clutter maps to decide when and where to apply MTI (moving target indication) for clutter suppression, or the use of polarization processing [2] to improve detection performance and circular polarization to mitigate rain clutter competing with targets when thunder storms are localized in nature. All of this was demonstrated in the AFRL Surveillance Facility in Rome NY in the 1980s.

Antenna

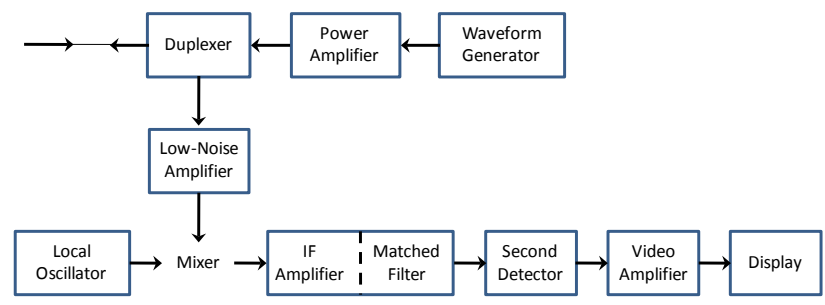

Fig. 3. Block Diagram Classical Radar Systems

This work was motivated in part by the fact that in clutter free regions, MTI causes loss of target detection performance, especially for slow or tangentially moving targets [3], and polarimetric waveforms are problematic in their own right for a similar reason. At this same time, work on knowledge based tracking [4] was under investigation by a number of researchers. While the level of sophistication of the earliest KB Tracker was primitive by today's standards, this early pioneering work established the foundation for applying nontraditional techniques to de-conflict detection and track declarations throughout the track formation and reporting process, as well as limiting the number of potential tracks associated with a set of measurement data (well before the application of particle filters to address this critical issue in multi-target tracking). The Expert System CFAR (constant false alarm rate) was developed to address the problems associated with optimum adaptive detection processing in realistic interference environments. No one CFAR detector performs best under all conditions. Many systems apply multiple CFAR detector algorithms simultaneously to address this problem. Historically, global decision making was left to the operator. In Expert System CFAR [5] we applied 11 simple rules and 4 CFAR detector algorithms (Cell Averaging, Greatest Of, Ordered Statistic, and Trimmed Mean) to achieve dramatically improved performance of 5 to $7 \mathrm{~dB}$ as discussed below and reported in the literature. This research naturally resulted in applications to STAP (Space Time Adaptive Processing). In Knowledge Based STAP, the proper selection of training data for covariance matrix estimation and the best selection of flight profiles from a "radar view" perspective is fundamental [6]. An extension to include map data, DTED, DFAD (busy highways, railways and rail traffic, presence of power lines, etc) in our knowledge based approach to parameter selection was developed and demonstrated in the 1990s. Eventually, the incorporation of adaptivity on transmit and receive was accomplished with the introduction of waveform diversity as an enabling technology [7]. With this, the loop was closed and full adaptivity on transmit and receive (including STAP filtering, CFAR detection and track processing) was accomplished; an essential ingredient to cognition was incorporated into radar. With full adaptivity, radar is well positioned to address the problems associate with spectral crowding in the radar bands. 


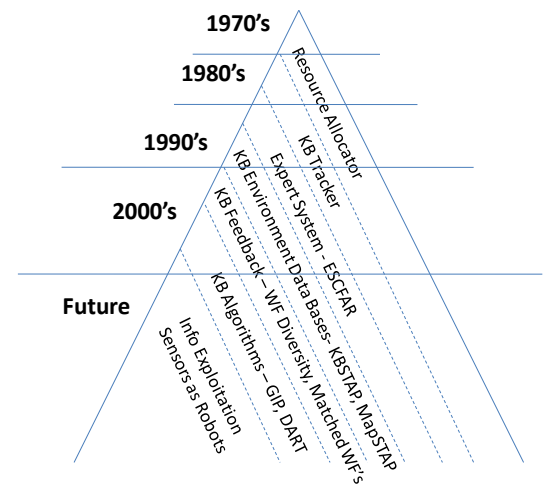

Fig. 4. Evolution of Intelligent Signal Processing Research at Rome

Next, we present more detail on two aspects of intelligent processing to set the groundwork for the next stage, cognitive processing.

\section{Expert System CFAR (Constant False Alarm Rate)}

The conflicting requirements for a high probability of detection and low probability of false alarm are rarely met in wide-area surveillance radar, due to spatial variations in the clutter. Any single algorithm is likely to be inadequate in a dynamically changing environment. The ES CFAR approach [8] is to select the filtering and CFAR algorithm(s) being executed at any one time based upon the observed characteristics of the interference. In reality, several STAP filter and CFAR detector algorithms are executed simultaneously. This requires sensing the environment, employing the most suitable filtering and detector algorithms, and applying a consensus algorithm to produce a global detection decision.

Figure 5 illustrates the ES-CFAR Processor, an Expert System with specific functions added to provide additional knowledge about interference, target detection and false alarm control.



Fig. 5. Expert System CFAR Processor

Background Analysis: As radar returns (data) are processed, the first task to be performed is determination of the statistical characteristics of the clutter. This entails identifying the probability density function (pdf) of the data as well as the associated parameters of the distribution using Ozturk's Algorithm [9].

Clutter Classification: Many STAP filter and CFAR algorithms are designed for detection processing in clutter behaving according to specific statistical distributions, excluding the effects of clutter discrete and outliers. This a priori knowledge provides the rule base which dictates the use of one CFAR algorithm over another in a given interference environment. Knowing the physical and statistical nature of the clutter and electromagnetic interference environment, combined with performance measures for various CFAR algorithms as a function of clutter type, aids in the selection of the most appropriate CFAR algorithm(s) [10].

Adaptive Filtering Library: Various forms of clutter suppression and Doppler filtering schemes are available [11] (MTI cancellers, Doppler filtering, space-time processing, etc.), but generally only one is used. Both the appropriate CFAR algorithm and filtering algorithm(s) must be applied.

CFAR algorithms: The relative performance of each CFAR algorithm must be quantified as a function of clutter type/statistic, detection probability, false alarm probability and CFAR processing loss. The library must contain CFAR algorithms with variable parameters such as Cell Averaging, Greatest-Of, Ordered Statistic, and Trimmed Mean. Detection performance is observed as a function of probability density function. Figure 6 is a plot of detection probability versus Weibull shape parameters for two CFAR algorithms. We can see the very different behavior of these algorithms under identical background conditions.



Fig. 6. Probability of Detection vs Weibull shape parameter for 2 CFAR Algorithms

Consensus Analysis: After selection of the most appropriate algorithms, detection processing is performed and decisions from the selected filter and CFAR algorithms must be weighted and fused to produce a satisfactory global detection decision. Here, the classical literature was used to provide algorithms and architectures for a working methodology to implement consensus processing [12]. 
Non Homogeneity Detector and Knowledge Based Space Time Adaptive Processing (KB-STAP)

The Non Homogeneity Detector [13] (NHD) is a logical extension of Trimmed Mean CFAR, and resulted from our interest in excising outliers from training data prior to covariance matrix estimation in STAP. In Trimmed Mean or TM CFAR, the excising of outliers is readily achieved by removing extremely large (clutter spikes) or small (clutter shadowed regions) returns after the square law stage of processing. As such, when dealing with scalar data, the difference between adjacent rank-ordered values provides for a natural discriminant. In STAP, the training data are vectors and not scalars. As such, there is no natural measure of similarity between two data vectors. In the NHD, this limitation is partially overcome by applying a STAP-like process via Hotelling's T-squared test, also called the Generalized Inner Product or GIP, producing a scalar measure of similarity, and then rank-ordering the output of this test. As in TM CFAR, the largest and smallest values are excised. Then, the training data vectors associated with those cells are dropped from the covariance matrix estimation process before a traditional STAP filter is applied for interference rejection and target detection. Similarly, a traditional STAP algorithm such as Sample Matrix Inversion (SMI), Modified SMI (MSMI), or the Generalized Likelihood Ratio Test (GLRT) may be applied for outlier detection and excision in place of the GIP test. The advantage in applying SMI or another STAP algorithm for NHD, is the beneficial consequence of having made target detection declarations which may then be further analyzed in the track stage of radar signal/data processing. Clutter returns, being stationary, are easily mitigated by the track processer.

Space-time adaptive processing (STAP) has been applied as a means for improving detection performance of airborne surveillance sensors. A very important, practical issue in fielding a STAP-based system concerns accurately estimating the interference covariance matrix and then computing an improved adaptive weight vector. Non-homogeneous interference, a feature of real-world airborne radar, complicates interference covariance matrix estimation and adaptive weight formulation [14].

The output of the space-time adaptive processor is

$$
y_{k}=w_{k}^{H} x_{k}
$$

where $W_{k}^{H}=s^{H} \hat{R}_{k}^{-1}$, and $s^{H}$ is the conjugate transpose of the target steering vector, $\hat{R}_{k}$ is the space-time interference covariance matrix estimate for $X_{k}$, which is the $M N x l$ complex signal vector for the $k t h$ range cell. In this case, $M$ equals the number of receive channels and $N$ indicates the number of coherent receive pulses.

A fundamental issue, evident from (1), concerns accurately estimating the true, unknown covariance matrix.
We typically estimate the covariance matrix via the minimum mean squared- error (MMSE) estimate as,

$$
\hat{R}_{k}=\frac{1}{P} \sum_{i=k-P / 2-G}^{k+P / 2+G} X_{i} X_{i}^{H} ; i \neq k-G, k, k+G,
$$

where $\mathrm{G}$ indicates the use of guard cells. The $X_{i}$ in (2) are referred to as secondary data. It is necessary that the $P$ secondary data vectors in (2) appear independent and identically distributed (iid) to the interference in the test cell for the MMSE estimate to be accurate. Nonhomogeneous interference violates this iid assumption.

The Non Homogeneity Detector (NHD) resulted from this interest in excising outliers from training data prior to covariance matrix formulation in STAP (see Figure 7).



Fig. 7. Multi-pass Non-homogeneity Detection Scheme [12]

In Trimmed Mean CFAR, the excising of outliers is readily achieved by removing extremely large (clutter spikes) or small (clutter shadowed regions) radar returns after the square law detector stage of processing. As such, when dealing with scalar data, the difference between adjacent rank-ordered values provides for a natural discriminant. In STAP, the training data are vectors and not scalars. As such, there is no natural measure of similarity between two data vectors. In the NHD, this limitation is overcome by applying a STAP like process via the GIP test, producing a scalar measure of similarity, and then rank-ordering the output of this test. As in Trimmed Mean CFAR, the largest and smallest values are excised. Then, the training data vectors associated with those cells are dropped from the covariance matrix estimation process before a traditional STAP filter.

We use measured airborne radar data from the MCARM Program to illustrate enhanced STAP capability resulting from the use of a nonhomogeneity detector (NHD). The postDoppler factored-time space (FTS) algorithm represents the specific MSMI STAP algorithm used in this analysis. Figure 8 compares the performance of the FTS algorithm with varied sample selection and adaptive weight computation. The top graph in Figure 8, labeled "FTS_SW", corresponds to the most commonly accepted approach for sample selection. In this case, a symmetric window (SW) about the test cell defines the secondary data to be used. Being similar to CACFAR, the basic idea behind this approach is that data most local to the test cell will appear most statistically similar. Notice in this scenario that the target at a range of 16.4 miles would go undetected with any reasonable threshold level. On the other hand, the bottom graph in Figure 8 shows the normalized adaptive filter output computed following the 
NHD stage of processing, and is akin to TM-CFAR. Observe that the target at a range of 16.4 miles is more clearly visible!
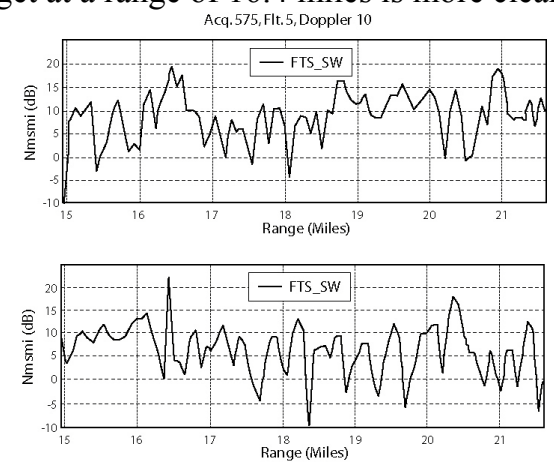

Fig. 8. MSMI test statistic versus range using SW method and GIP and SMI-based NHD schemes

When applied to a full scene of measured multichannel airborne radar data under a knowledge based controller, the effect of NHD screening of training data on detection performance is dramatic, as further illustrated in Figure 9 below.

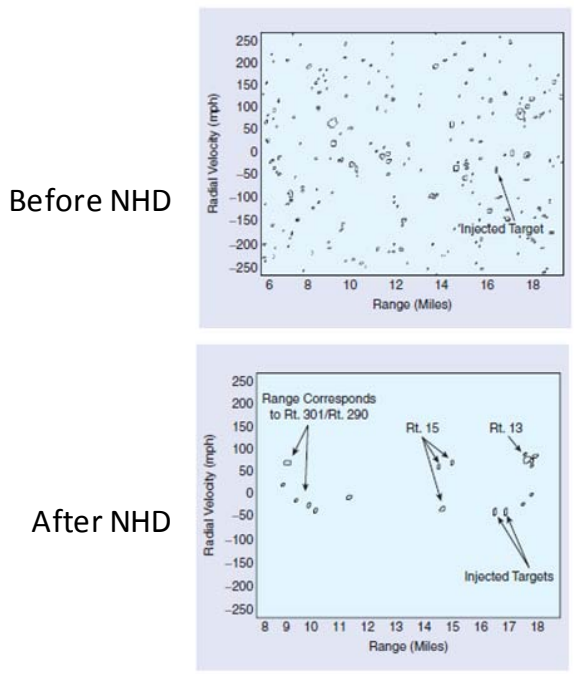

Fig. 9. Target declarations before and after applying the NHD [14]

Over Resolved Target Parameter Estimation in the Presence of Clutter and Electromagnetic Interference

Targets and interference are resolved in range (using wide bandwidth) and in cross-range (using long integration periods and Doppler processing). Analysis of the multiple returns from a single extended target can provide improved performance in terms of interference rejection, detection and parameter estimation. A technique has been developed that achieves significantly better performance for extended targets in clutter and electromagnetic interference. This algorithm assumes some knowledge of the target of interest and its orientation. A coherent template is employed to integrate energy from across the extended target return. Where the target size and orientation are known perfectly this algorithm is straight forward and deterministic.

When the knowledge of the target is limited the algorithm becomes more complex. Multiple coherent templates are applied to the data and the GIP applied to the multiple analyses, including iteration over the parameters space of the templates. Selection of the initial and iterative templates by cognitive processing will greatly extend the application of this algorithm, even to cases where there is very limited prior knowledge concerning targets.

The template integration step in this algorithm uses the GIP based approach. For example, consider a circular-shaped object on the ground. This object is a conductive pipe, i.e., the cross-section of the example object is a circle. Its location (slant range, azimuth) and radius are unknown [15].

An L-band radar with a bandwidth of $250 \mathrm{MHz}$ was modeled. The platform altitude was 600 meters and the platform velocity was 50 meters/second.



Fig.10. GIP as a function of slant range

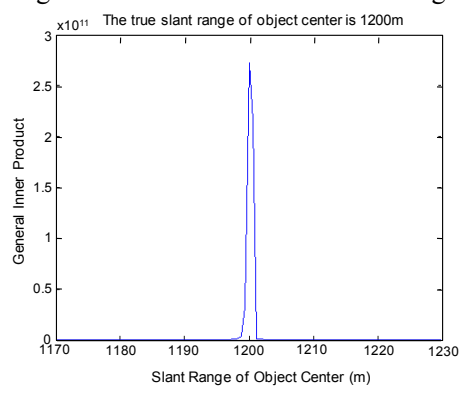

Fig.11. GIP as a function of radius

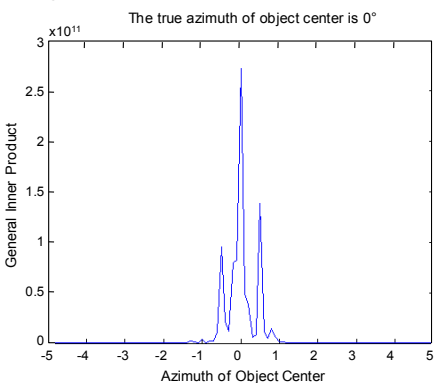

Fig.12. GIP as a function of azimuth

Case 1: Assume the radius and azimuth in the GIP detector match those of the object. Figure 10 shows the GIP output as a function of slant range. The GIP sharply peaks at the true range of object.

Case 2: Assume the range and azimuth of the object center in the detector match those of the object. Figure 11 shows the GIP output as a function of the radius of the ring object.

Case 3: Assume the radius of the ring object and its center range in the detector match the true values. Figure 12 shows the GIP output as a function of the azimuth angle of the ring center. 


\section{ONE PossibLE VISION OF COGNITIVE RADAR}

The Adaptive and intelligent (expert system, knowledgebased, knowledge-aided) processing algorithms were developed by emulating what the experts in each area of radar processing would have done in order to optimize performance of their individual area (filtering, CFAR detection, track processing, declaration and discrimination). As the number of intelligent processing areas and algorithms has increased (Figure 4), the number of areas of technical expertise has increased as well. The interaction between these important areas cannot be fully addressed by adaptive and intelligent processing alone. A further step (cognitive processing) is required.

Figure 12 illustrates the essential components of a futuristic radar for operation in spectrally congested environments. A confluence of factors must come together to meet the requirements identified above. The need for autonomous operations within the context of remote sensing demands that both platform and radar be employed intelligently. Spectral awareness is a critical component of cognitive radar. In order to operate dynamically within geographically and temporally defined spectrum limitations, a number of challenges in spectrum management must be overcome.

Adaptive algorithms (STAP, CFAR, GIP) and intelligent algorithms (expert system, knowledge-aided, etc.) will be employed in a number of functional areas in this diagram. But that by itself cannot achieve the desired capability. The red lines in Figure 13 indicate interactions between the individual functions that could lead to further performance improvement if employed effectively. Development of this very complex and integrated processing approach will require a major step forward in radar signal and data processing - Cognition.

\section{Summary}

The ever increasing demand on remote sensing capabilities directly conflicts with the accelerating loss of spectrum allocation. Increased spectral awareness and waveform diversity can be applied to this problem through cognitive processing and control of modern radar. This paper motivates the development of essential technology for this purpose.

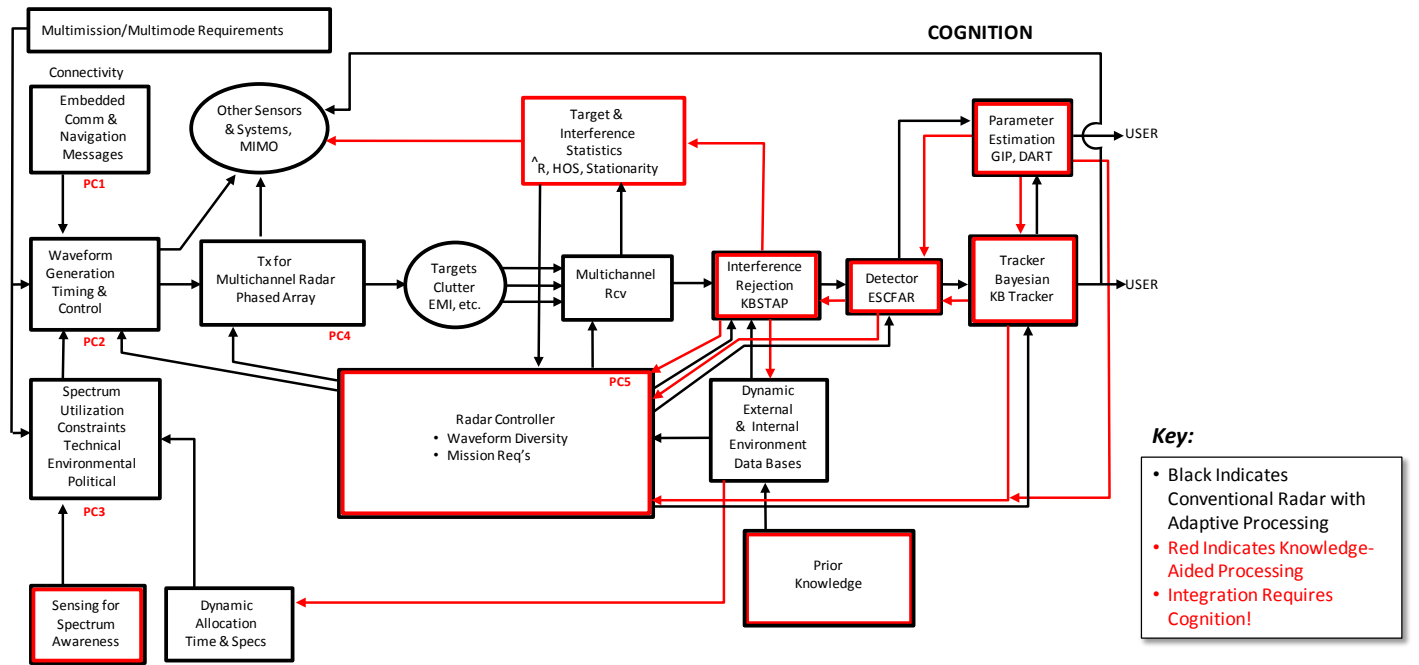

Fig.13. Cognitive Radar

\section{REFERENCES}

[1] V. Vannicola, "Expert system for sensor resource allocation," in Proc. $33^{\text {rd }}$ Midwest Symposium of Circuits and Systems, Aug 1990, pp.10051008 .

[2] M. Wicks, R. Brown, V. Vannicola, "Polarization Radar Processing Technology," in Proc. IEEE International Radar Conference, May 1990, Washington DC. pp. 409 - 416.

[3] V. Vannicola, R. Brown, M. Wicks, "Area MTI - A Multiple Scan Signal Processing Technique," in Proc SPIE, 1989.

[4] A. Crobeil and L. Hawkins, 1991, "Knowledge-based tracker assessment program," Final Technical Report, RL-TR-91-73, 1992

[5] W. Baldygo, R. Brown, P. Antonik, G. Capraro, L. Hennington, "Artificial Intelligence Applications to Constant False Alarm Rate (CFAR) Processing," in Proc. IEEE National Radar Conference, April 1993, pp. 275-280.

[6] M. Wicks, W. Melvin, P. Chen, "An efficient architecture for nonhomogeneity detection in space-time adaptive processing airborne early warning radar," in Proc. IEE International Radar Conference, Oct 1997 , pp. $295-299$.
[7] V. Amuso, S. Blunt, E. Mokole, R. Schneible, M. Wicks, Principles of Waveform Diversity and Design, $1^{\text {st }}$ ed., Sci-Tech Publishing, Raleigh, NC, TBD.

[8] M.C. Wicks, W.J. Baldygo, R.D. Brown, "Expert system constant false alarm rate (CFAR) processor," U.S. Patent 5499 030, March 121996.

[9] S. Salerno, "A Matlab-based ozturk algorithm implementation," AFRL-IF-RS-TM-2006-4 In-house Tech. Memorandum, 2006.

[10] M. Slamani, "A new approach to radar detection based on the partitioning and statistical characterization of the surveillance volume," Doctoral thesis, Syracuse University, Syracuse, NY, 1994.

[11] G. Capraro, A. Farina, H. Griffiths, M. Wicks, "Knowledge-Based Radar Signal and Data Processing," IEEE Signal Processing Magazine, vol 23, Jan. 2006, pp. 18-29.

[12] P. Varshney, Distributed Detection and Data Fusion, Springer-Verlag, 1997.

[13] W. Melvin, M. Wicks, P. Chen. "Nonhomogeneity detection method and apparatus for improved adaptive signal processing," US Patent 5 706 013, Jan. 6, 1998.

[14] M. Wicks, M. Rangaswamy, R. Adve, T. Hale, "Space-time adaptive processing, a knowledge based perspective for airborne radar," IEEE Signal Processing Magazine, vol 23, pp. 51-65, Jan. 2006

[15] M. Wicks, Y. Zhang, "Optimized detection of spatially extended fixed objects in clutter," in Proc IEEE RadarCon09, May 2009, pp. 1-6. 